\title{
A Fair Comparison Between OMA and NOMA For Cell-Free Massive MIMO Systems
}

\author{
Ruan Failache, Aline Ohashi, André Fernandes, Roberto Rodrigues, André Cavalcante and João Weyl
}

\begin{abstract}
Non-orthogonal multiple access (NOMA) can improve the performance of cell-free massive MIMO systems by reducing the effects of pilot contamination. To assess NOMA performance most of the literature compares it with orthogonal multiple access (OMA). However, these works comparisons are unfair. From the authors' perspective, a fair comparison should consider the same number of users and pilots for NOMA and OMA. In this work, we evaluate these multiple access schemes under our envisioned fair terms. Our results show that NOMA provides gains of user spectral efficiency (SE) and aggregate sumrate superior to OMA in normal and stressed scenarios.
\end{abstract}

Keywords - Cell-free massive MIMO, fair comparison, NOMA, OMA, pilot contamination.

\section{INTRODUCTION}

The ever increasing demand requirements for mobile data traffic due to new applications, like the Internet of Things (IoT), can be fulfilled by the fifth generation (5G) and beyond in two ways: (1) increasing the bandwidth and (2) using the bandwidth more efficiently. One way to achieve the former is increasing the operating carrier frequency, where more band is available. However, such solution is not an easy task, once it finds barriers as hardware development, modifications in the technology to be compatible in these frequencies, besides that, they need licensing these frequency bands, which can be costly prohibitive. Thus, the efficient use of bandwidth appears as the most natural direction. Two ways to achieve the latter are cellfree massive multiple-input multiple-output (MIMO) networks and non-orthogonal multiple access (NOMA) [1]-[4].

Cell-free massive MIMO networks consist of a communication system where each user equipment (UE) is served by a group of access points (APs) in a user-centric fashion, resulting in macro-diversity gain and a more uniformly distributed user's rate over coverage area. Despite this, these networks may not scale well with the number of users once the channel estimation of each user needs an orthogonal pilot sequence, which are a limited resource on the network. Repeat sequences between a group of users may overcome this problem, but it generates pilot contamination, i.e. coherent interference between users with the same pilot sequence. Centralized massive MIMO

Ruan Failache ${ }^{1}$, Aline Ohashi ${ }^{1}$, André Fernandes ${ }^{1}$, Roberto Rodrigues $^{1}$, André Cavalcante ${ }^{2}$ and João Weyl', ${ }^{1}$ Applied Electromagnetism Laboratory (LEA), Federal University of Pará (UFPA), Belém-PA, Brazil. ${ }^{2}$ Ericsson Research, Ericsson Telecomunicações S.A., Brazil. E-mails: \{ruan.failache\}@itec.ufpa.br, \{alineohashi;andrelpf;rmr;jweyl\}@ufpa.br, andre.mendes.cavalcante@ericsson.com. This work was supported partial by the Innovation Center, Ericsson Telecomunicações S.A., Brazil, by the National Council for Scientific and Technological Development (CNPq) and by the Coordination of Superior Level Staff Improvement (CAPES). avoid this by taking advantage of cell structure, which is not the case of cell-free networks [1], [2].

Previous works [3]-[5] indicated that pilot contamination problems could be circumvented using the power-domain NOMA technique. This technique performs different power allocation levels based on the channel conditions. The basic idea is to superimpose the user's signals, allocating more power to users with the worst channel condition and less power to users with the best ones, balancing the achieved sum rate. Moreover, through successive interference cancellation (SIC), the superimposed signals can be separated at the UE [6].

Most of the cell-free massive MIMO-NOMA works compare it with the orthogonal multiple access (OMA) technique. Nonetheless, those techniques comparisons in general are unfair from the authors' perspective since they do not consider the same number of users served and the pilot sequence reuse in both scenarios. A fair comparison should consider the same number of users and cope with pilot contamination. To the best of the author's knowledge, the only work to indirectly address this kind of fair comparison in the context of cell-free massive MIMO-NOMA was [7]. However, this work do not quantifies the performance differences between fair and unfair comparisons.

In this way, the present work analyzes the performance differences between fair and unfair comparisons between OMA and NOMA in cell-free massive MIMO networks. The performance is evaluated in terms of user spectral efficiency (SE) and aggregate sum rate. To this end, we have adapted a signal model for cell-free massive MIMO networks to be compatible with both OMA and NOMA under our assumed fair comparison.

The remainder of this paper is divided into four sections. Section II presents the considered system, channel, and signal models. Section III outlines the calculations for the achievable rates. Section IV shows the adopted scenario, the assumptions, and the obtained results. Finally, Section V summarizes the main conclusions.

Notations: Lower-case bold and upper-case bold denote vectors and matrices, respectively. The superscripts ()$^{H},()^{T}$, and ()$^{*}$ denote the conjugate-transpose, transpose and conjugate, respectively. The expectation operator is denoted by $\mathbb{E}\{\cdot\}$ and $\mathcal{C N}\left(0, \sigma^{2}\right)$ stands for a complex Gaussian vector with zero mean and variance $\sigma^{2}$.

\section{System Functionality}

\section{A. System and Channel Models}

We assume a downlink (DL) transmission of both OMA and NOMA cell-free massive networks, composed of $M$ spatially 
distributed single-antenna APs serving $K$ single-antenna users under time division duplex (TDD) protocol in the same timefrequency resource block. We also assume that the users are grouped in $N$ clusters, which will utilize the same pilot sequence [3]. By exploiting the reciprocity in TDD, we model both the DL and uplink (UL) physical propagation channel between the AP $m \in\{1, \ldots, M\}$ and the user $k \in\{1, \ldots, K\}$ in the cluster $n \in\{1, \ldots, N\}$ as

$$
h_{m n k}=\sqrt{\beta_{m n k}} \tilde{h}_{m n k}
$$

where $\beta_{m n k}$ and $\tilde{h}_{m n k}$ denote the large-scale fading and smallscale fading, respectively. The components of $\tilde{h}_{m n k}$ are independent and identically distributed (i.i.d.) $\mathcal{C N}(0,1)$ random variabless (RVs) [5]. Futhermore, the APs are connected to a central processing unit (CPU) via a perfect and error-free fronthaul with unlimited capacity.

\section{B. Uplink Channel Estimation}

By exploiting the reciprocity in TDD, the DL channel estimate can be obtained from UL channel estimation. The latter is easily made with the transmission of orthogonal pilot sequences by each cluster's users. Aiming to provide a fair comparison between OMA and NOMA, where the former also suffers from pilot contamination, we assumed the same pilot's assignment to the users within the same cluster, i.e., $\boldsymbol{\varphi}=\left[\boldsymbol{\varphi}_{1}, \ldots, \boldsymbol{\varphi}_{n}, \ldots, \boldsymbol{\varphi}_{\boldsymbol{N}}\right] \in \mathbb{C}^{\tau_{p} \times N}$, where $\left\|\boldsymbol{\varphi}_{n}\right\|^{2}=1$. Thereby, the signal received at the $m$-th AP during the UL channel estimation can be expressed as

$$
\mathbf{y}_{p, m}=\sqrt{\tau_{p} \rho_{u}} \sum_{n=1}^{N} \sum_{k=1}^{K_{n}} h_{m n k} \boldsymbol{\varphi}_{n}+\mathbf{w}_{m}
$$

where $\tau_{p}, \rho_{u}$ and $\varphi_{n}$ denotes the pilot sequence length (in samples), UL normalized signal-to-noise (SNR), and the pilot sequence assigned to the $n$-th cluster, respectively. The elements of $\mathbf{w}_{m}$ are i.i.d. $\mathcal{C N}(0,1) \mathrm{RVs}$, and $K_{n}$ denotes the number of users per cluster. In this way, the received pilot signal from the $m$-th AP at the $n$-th cluster is projected over the pilot sequence $\varphi_{n}$, providing

$$
\tilde{y}_{p, m n}=\varphi_{n}^{H} \mathbf{y}_{p, m}=\sqrt{\tau_{p} \rho_{u}} \sum_{k=1}^{K_{n}} h_{m n k}+\varphi_{n}^{H} \mathbf{w}_{m} .
$$

Similar to [3], we employ the minimum mean square error (MMSE) channel estimation, given by

$$
\hat{h}_{m n k}=\frac{\sqrt{\tau_{u} \rho_{u}} \beta_{m n k}^{\mathrm{ul}}}{\tau_{u} \rho_{u} \sum_{k=1}^{K_{n}} \beta_{m n k}^{\mathrm{ul}}+1} \tilde{y}_{p, m n}
$$

where $\tau_{u}$ is the samples in the coherence interval used for UL transmission. Finally, taking the variance of the UL channel estimation, we obtain

$$
\gamma_{m n k}=\mathbb{E}\left\{\left|\hat{h}_{m n k}\right|^{2}\right\}=\frac{\tau_{p} \rho_{u}\left(\beta_{m n k}^{\mathrm{ul}}\right)^{2}}{\tau_{u} \rho_{u} \sum_{k^{\prime}=1}^{K_{n}} \beta_{m n k^{\prime}}^{\mathrm{ul}}+1} .
$$

\section{Downlink Data Transmission}

In this phase, due to reciprocity, we assume the same channel as the estimated in the UL phase. For each cluster $n$, the superimposed signal transmitted to the $K_{n}$ users can be written as

$$
q_{n}=\sum_{k=1}^{K_{n}} \sqrt{P_{n k}} q_{n k},
$$

where $q_{n k}$ is the transmitted symbol for $k$-th user at the $n$ th cluster, and $P_{n k}=\sum_{m=1}^{M} \eta_{z m n k}$ is the power of the $k$-th user at the $n$-th cluster. The former is a Gaussian RV with zero-mean and unit variance. The $\eta_{m n k}$ in the latter is the power control coefficient for each AP to serve each user of each cluster, being defined for OMA and NOMA, respectively, as

$$
\eta_{z m n k}=\left\{\begin{array}{c}
\frac{\rho_{d}}{\gamma_{m n k^{\prime}}}, \text { if } z=0, \\
\frac{\rho_{d} \alpha_{n k}}{\left(\sum_{k^{\prime}=1}^{K} \gamma_{m n k^{\prime}}\right)}, \text { if } z=1,
\end{array}\right.
$$

where $\alpha_{n k}$ denotes the power allocation coefficient associated with the power-domain NOMA scheme for the users in the same $n$-th cluster, and $z \in[0,1]$ is the adopted multiple access scheme, being 0 for OMA and 1 for NOMA. Therefore, the received signal at the $k$-th user in the $n$-th cluster can be expressed, respectively, for OMA and NOMA as [3]

$$
r_{z n k}=\mathbf{u}_{z n k}^{T} \mathbf{h}_{n k}^{\mathrm{dl}} q_{n}+\sum_{n^{\prime} \neq n}^{N} \mathbf{u}_{z n^{\prime} k}^{T} \mathbf{h}_{n k}^{\mathrm{dl}} q_{n^{\prime}}+w_{n k},
$$

where $w_{n k} \sim \mathcal{C N}(0,1), \mathbf{u}_{n}$, and $\mathbf{h}_{n k}^{\mathrm{dl}}$ denotes the receiver thermal noise, precoding vector for the $n$-th cluster and the DL channel of $k$-th user at $n$-th cluster, respectively. Assuming matched filter (MF) precoding, $\mathbf{u}_{z n k}$ can be expressed as

$$
\mathbf{u}_{z n k}=\left[\eta_{z 1 n k}^{1 / 2} \hat{h}_{1 n k}^{*}, \cdots, \eta_{z m n k}^{1 / 2} \hat{h}_{m n k}^{*}\right]^{T} .
$$

Let $\hat{q}_{n k}$ be the estimation of $q_{n k}$ in such a way that $q_{n k}$ and $\hat{q}_{n k}$ are jointly Gaussian distributed with a normalized correlation coefficient $\rho_{n k}$, which can be depicted as follows

$$
q_{n k}=\rho_{n k} \hat{q}_{n k}+e_{n k},
$$

where $\rho_{n k}=1 / \sqrt{1+\sigma_{e_{n k}}^{2}}$ is related to the quality of the channel state information (CSI) estimation, and hence direct associated to the severity of SIC imperfection, $e_{n k} \sim$ $\mathcal{C N}\left(0, \sigma_{e_{n k}}^{2} /\left[1+\sigma_{e_{n k}}^{2}\right]\right)$ is the estimation error and $\hat{q}_{n k} \sim$ $\mathcal{C N}(0,1)$. Beyond that, $\hat{q}_{n k}$ and $e_{n k}$ are assumed to be statistically independent [3].

\section{Achievable Sum Rate}

The exact user's rate of wireless systems limited by interference is generally unknown, but convenient lower bounds can be obtained. In this way, the use-and-then-forget (UatF) bound has arisen as an efficient way to obtain tight achievable DL rate in massive MIMO scenarios [8], [9]. Thus, the user's rate is given by

$$
R_{z n k}^{\mathrm{UatF}}=\phi \log _{2}\left(1+\mathrm{SINR}_{z n k}\right),
$$


where $\phi=\left(\tau_{c}-\tau_{p}\right) / \tau_{c}$ is the pre-log factor, $\tau_{c}$ is the coherence interval, and $\mathrm{SINR}_{z n k}$ is the effective signal-to-interferenceplus-noise-ratio (SINR) for the $k$-th user at the $n$-th cluster, composed by desired and interference signals, which can be written as (12) at the top of next page.

The terms $\mathbb{E}\left\{\left|\mathrm{DS}_{n k}\right|^{2}\right\}, \quad \mathbb{E}\left\{\left|\mathrm{SI}_{n k}\right|^{2}\right\}, \quad \mathbb{E}\left\{\left|\mathrm{IC}_{n k}^{\mathrm{OMA}}\right|^{2}\right\}$, $\mathbb{E}\left\{\left|\mathrm{IC}_{n k}^{\mathrm{NOMA}}\right|^{2}\right\}, \mathbb{E}\left\{\left|\mathrm{OC}_{n k}\right|^{2}\right\}$ and $\mathbb{E}\left\{\left|\mathrm{RI}_{n k}\right|^{2}\right\}$ in Equation (12), are the powers of the desired signal, self-interference, inside cluster inter-user OMA interference, inside cluster inter-user NOMA interference, outside cluster inter-user interference, and residual interference due to SIC, respectively. We detailed the mentioned terms in the following subsections.

\section{A. Computation of $D S_{n k}$}

The desired signal can be derived from [10]

$$
\mathrm{DS}_{n k}=\mathbb{E}\left\{\boldsymbol{u}_{z n k}^{T} \boldsymbol{h}_{n k}^{\mathrm{dl}}\right\} q_{k} .
$$

Defining the channel estimation error by $\varepsilon_{m n k}=h_{m n k}^{\mathrm{ul}}-$ $\hat{h}_{m n k}^{\mathrm{ul}}$, which is a gaussian distribution with zero mean, the mean of the product between $\varepsilon_{m n k}$ and any independent gaussian RV will be zero. Thus, it yields

$$
\mathbb{E}\left\{\left|\mathrm{DS}_{n k}\right|^{2}\right\}=\sum_{m=1}^{M} \eta_{z m n k} \gamma_{m n k}^{2}
$$

\section{B. Computation of $S I_{n k}$}

The self-interference can be obtained from [10]

$$
\mathrm{SI}_{n k}=\left(\boldsymbol{u}_{z n k}^{T} \boldsymbol{h}_{n k}^{\mathrm{dl}}-\mathbb{E}\left\{\boldsymbol{u}_{z n k}^{T} \boldsymbol{h}_{n k}^{\mathrm{dl}}\right\}\right) q_{k} .
$$

Using a similar procedure as of the desired signal derivation, we have

$$
\mathbb{E}\left\{\left|\mathrm{SI}_{n k}\right|^{2}\right\}=\sum_{m=1}^{M} \eta_{z m n k} \beta_{m n k} \gamma_{m n k}
$$

\section{Computation of $I C_{n k}^{O M A}$}

The inside cluster inter-user OMA interference is related to the OMA users in the same cluster and can be derived from the inter-user interference as [9]

$$
\mathbb{E}\left\{\left|\mathrm{IC}_{n k}^{\mathrm{OMA}}\right|^{2}\right\}=\sum_{m=1}^{M} \beta_{m n k} \sum_{k^{\prime}=1, k^{\prime} \neq k}^{K} \eta_{z m n k^{\prime}} \gamma_{m n k^{\prime}} .
$$

\section{Computation of $I C_{n k}^{N O M A}$}

Similarly, the inside cluster inter-user NOMA interference can be obtained from the inter-user interference as

$$
\mathbb{E}\left\{\left|\mathrm{IC}_{n k}^{\mathrm{NOMA}}\right|^{2}\right\}=\sum_{m=1}^{M} \beta_{m n k} \sum_{k^{\prime}=1}^{k-1} \eta_{z m n k^{\prime}} \gamma_{m n k^{\prime}} .
$$

\section{E. Computation of $O C_{n k}$}

This interference is related to the users outside the cluster $n$, which have different pilot sequences from the interested user, characterizing an inter-user non-coherent interference [9], [10]

$$
\mathbb{E}\left\{\left|\mathrm{OC}_{n k}\right|^{2}\right\}=\sum_{m=1}^{M} \beta_{m n k} \sum_{n^{\prime}=1, n^{\prime} \neq n}^{N} \sum_{k^{\prime}=1}^{K} \eta_{z m n^{\prime} k^{\prime}} \gamma_{m n^{\prime} k^{\prime}} .
$$

\section{F. Computation of $R I_{n k}$}

Based on [3], [5], [10], the residual interference can be derived as follows

$$
\mathrm{RI}_{n k}=\sum_{k^{\prime \prime}=k+1}^{K} \boldsymbol{h}_{n k}^{\mathrm{dl} T} \boldsymbol{u}_{z n k^{\prime \prime}} q_{n k^{\prime \prime}}-\mathbb{E}\left[\boldsymbol{h}_{n k}^{\mathrm{dl} T} \boldsymbol{u}_{z n k^{\prime \prime}}\right] \hat{q}_{n k^{\prime \prime}} .
$$

Let $\varepsilon_{m n k} \triangleq h_{m n k}-\hat{h}_{m n k}$ be channel estimation error for the $k$-th user of the $n$-th cluster to the $m$-th AP. Knowing that the variance of the sum of independent RVs is equal to the sum of their variances, we have [5]

$$
\begin{aligned}
& \mathbb{E}\left\{\left|\mathrm{RI}_{n k}\right|^{2}\right\}= \sum_{k^{\prime \prime}}=k+1 \\
& \underbrace{K}_{2^{\circ} \text { Term }}[\underbrace{\mathbb{E}\left\{\mid \mathbb{E}\left[\left.\boldsymbol{h}_{n k}^{\mathrm{dl} T} \boldsymbol{u}_{z n k^{\prime \prime}} q_{n k^{\prime \prime}}\right|^{2}\right\}\right.}_{1^{\circ} \text { Term }}-
\end{aligned}
$$

Making some algebraic operations, it follows that the first term can be written as

$$
\mathbb{E}\left\{\left|\boldsymbol{h}_{n k}^{\mathrm{dl}}{ }^{T} \boldsymbol{u}_{z n k^{\prime \prime}} q_{n k^{\prime \prime}}\right|^{2}\right\}=\sum_{m=1}^{M} \eta_{z m n k^{\prime \prime}}\left(\gamma_{m n k}^{2}+\gamma_{m n k} \beta_{m n k}\right) \text {. }
$$

Knowing that the mean of the product between $\varepsilon_{m n k}$ and any independent gaussian RV equals zero and making some algebraic operations, the second term can be rewritten as

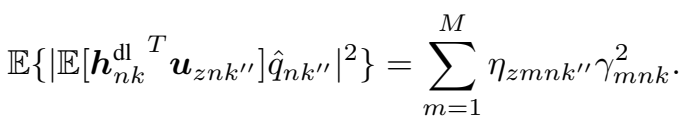

By substituting (22) and (23) into (21), it yields the final expression

$$
\mathbb{E}\left\{\left|\mathrm{RI}_{n k}\right|^{2}\right\}=\sum_{k^{\prime \prime}=k+1}^{K} \sum_{m=1}^{M} \eta_{z m n k^{\prime \prime}} \gamma_{m n k} \beta_{m n k} .
$$

\section{Simulation Scenario And Results}

We modeled the scenario as a cell-free massive MIMONOMA system made of 100 APs and 20 UEs, both with single antennas. The APs and UEs are distributed uniformly over an area of $1 \mathrm{~km} \times 1 \mathrm{~km}$, and we set the coherence interval as $\tau_{c}=196$ samples [3], [7]. For a fair comparison, both OMA and NOMA are grouped in $N$ clusters of 2 UEs so that the pre$\log$ factor for both cases is $\phi_{O M A}=\phi_{N O M A}=\left(\tau_{c}-N\right) /\left(\tau_{c}\right)$.

We assume that each user's cluster had a pre-allocated pilot sequence that was used to estimate the channels. Thus both NOMA and OMA will support the same number of UEs and being affected by pilot contamination. The propagation model adopted is the 3GPP Urban Micro (UMi) path-loss model defined in 3GPP TR 38.901, where $\beta_{m n k}$ follows a log-normal shadowing fading distribution [11]. In Table I, we present the basic simulation parameters [3], [10]:

To investigate the performance differences under fair and unfair comparisons under OMA and NOMA, Fig. 1 presents the cumulative distribution function (CDF) versus the user SE for two thousand Monte Carlo realizations. Two cases of 


$$
\operatorname{SINR}_{z n k}=\frac{\mathbb{E}\left\{\left|\mathrm{DS}_{n k}\right|^{2}\right\}}{\mathbb{E}\left\{\left|\mathrm{SI}_{n k}\right|^{2}\right\}+(1-z) \mathbb{E}\left\{\left|\mathrm{IC}_{n k}^{\text {OMA }}\right|^{2}\right\}+z \mathbb{E}\left\{\left|\mathrm{IC}_{n k}^{\mathrm{NOMA}}\right|{ }^{2}\right\}+\mathbb{E}\left\{\left|\mathrm{OC}_{n k}\right|^{2}\right\}+z \mathbb{E}\left\{\left|\mathrm{RI}_{n k}\right|^{2}\right\}}
$$

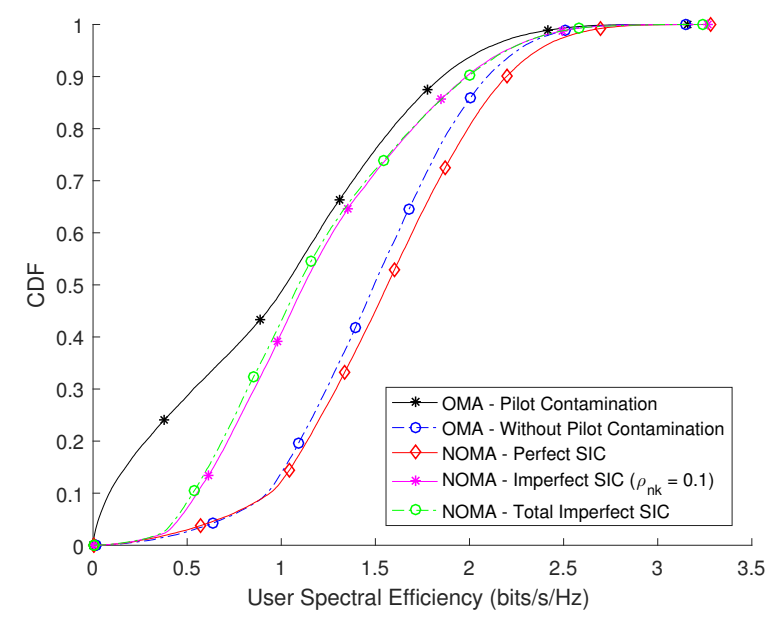

(a) $K=20$

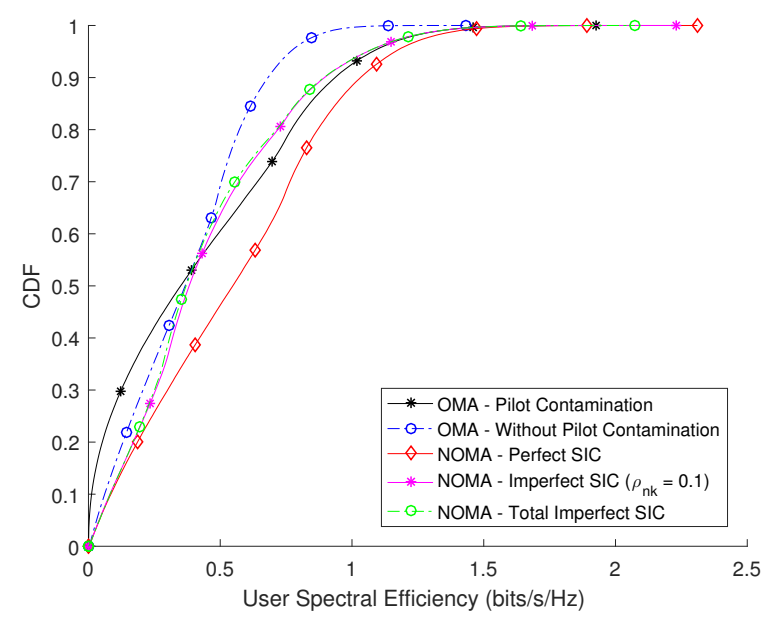

(b) $K=100$

Fig. 1: CDF's comparison of user SE between OMA versus NOMA for (a) $K=20$ UEs and (b) $K=100$ UEs, considering imperfect SIC of $\rho_{n k}=0.1$ where $M=100$ and $\tau_{p}=N=10$. For NOMA, the power's allocation is the same for each cluster obeying the rate of $3: 7 \forall n$ [3].

TABLE I: SIMULATION SETTINGS

\begin{tabular}{|c|c|}
\hline Parameter & Value \\
\hline Number of APs, $M$ & 100 \\
\hline Number of UEs, $K$ & 20,100 \\
\hline Carrier frequency & $1.9 \mathrm{GHz}$ \\
\hline Bandwidth & $20 \mathrm{MHz}$ \\
\hline RX noise figure & $100 \mathrm{~dB}$ \\
\hline UL Transmit power, $\rho_{\mathrm{u}}$ & $200 \mathrm{~mW}$ \\
\hline DL Transmit power, $\rho_{\mathrm{d}}$ & $10 \mathrm{~m}, 1.65 \mathrm{~m}$ \\
\hline
\end{tabular}

number of users were illustrated: (a) $K=20$ and (b) $K=$ 100. Five cases of multiple access schemes are considered for each number of users: NOMA with perfect SIC, NOMA with imperfect SIC $\left(\rho_{n k}=0.1\right)$, NOMA with total imperfect SIC, OMA with pilot contamination, and OMA without pilot contamination (each cluster has just one user). The chosen value for imperfect SIC (0.1) reflects an imperfection of $90 \%$ in the SIC process, which has been commonly adopted in previous works [3], [5].

In Fig.1 (a), we verify that under ideal conditions, without pilot contamination and perfect SIC, NOMA provides a slightly better user SE than OMA for most users, except for the $10 \%$ worst likely users, where their SE equates. In a fair comparison, with the same number of pilots (which in OMA stands for the case of pilot contamination), we observe that the user SE gains obtained in NOMA are more significant than OMA, even in the presence of total imperfect SIC. We also verify that pilot contamination in OMA affects mainly the $40 \%$ worst likely users, where user SE reductions up to $0.85 \mathrm{bits} / \mathrm{s} / \mathrm{Hz}$ can be observed. When we stress the network considering
$K=100$ UEs, i.e, Fig. 1 (b), we observe that NOMA is better than OMA for all users under ideal conditions. Besides, in a fair comparison, NOMA with imperfect SIC presents a better SE than OMA pilot contamination for the $55 \%$ worst likely users. However, OMA presents gains over NOMA for the best likely users. As one of the main goals of the cell-free massive MIMO network is to increase user's rate fairness on the network, we can say that in a fair comparison, NOMA still outperforms OMA even in stressed systems.

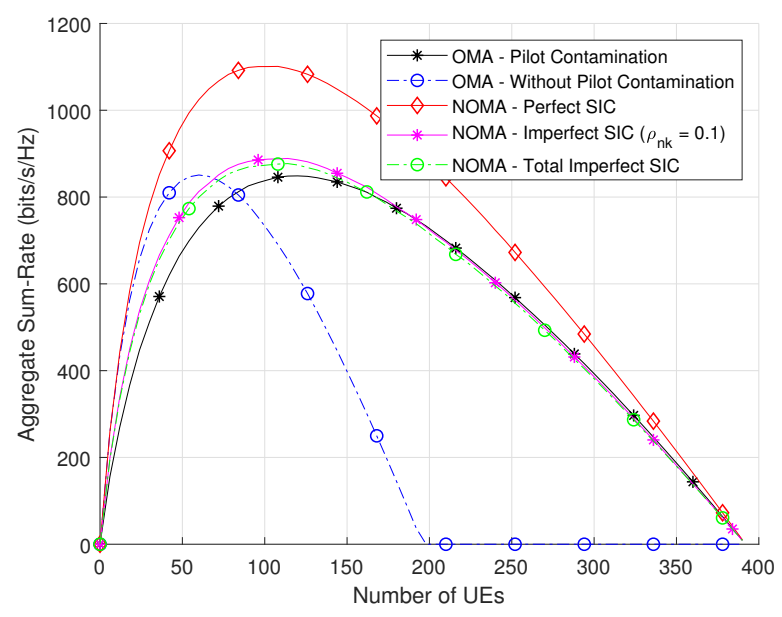

Fig. 2: Aggregate sum-rate per-user comparison between OMA and NOMA by number of UEs.

To better investigate the effects of the number of UEs in our considered multiple access schemes, Fig. 2 presents a sensibility analysis of the aggregated sum-rate versus the 
number of UEs for $M=100$ APs. Under the perspective commonly addressed in the literature, that we consider as unfair, OMA has better aggregated sum-rate up to 72 UEs in relation to NOMA with imperfect SIC. Such behavior occurs due to the pre-log factor and the non-consideration of pilot contamination. In a fair comparison, with the same number of UEs and pilots, we observe that the aggregated sum-rate obtained in NOMA is better than OMA up to 150 UEs. Both aggregate sum-rates start to behave similarly since from that value. However, NOMA probably still is a better alternative than OMA in these extreme cases, once our previous results showed that NOMA has better user's rate fairness than OMA.

\section{CONCLUSION}

This work presented a fair performance analysis comparison between OMA and NOMA operation for cell-free massive MIMO networks in terms of SE and aggregate sum rate. We assumed the same number of UEs and orthogonal pilots for both OMA and NOMA (a fair performance comparison), which is an approach different than the one generally considered for OMA in the literature of cell-free massive MIMO operating under NOMA. The obtained results indicate that the performance of OMA as generally considered in the literature (an unfair approach) is super-estimated, with bigger SE and aggregate sum-rate over NOMA when fewer UEs are considered. The results also indicate that under a fair comparison NOMA outperforms OMA both in normal and stressed scenarios (i.e., with the number of UE closer or bigger than the number of AP). Furthermore, if SIC imperfection approaches zero, i.e. perfect SIC, NOMA achieved better rates even when compared to OMA without pilot contamination. In future works, we plan to consider more optimal pilot assignment strategies for NOMA, which implicates an optimal clusterization of users. In addition to an optimized power allocation of users performing NOMA.

\section{REFERENCES}

[1] G. Interdonato, E. Bjornson, H. Q. Ngo, P. Frenger, and E. G. Larsson, "Ubiquitous cell-free massive MIMO communications," EURASIP J. Wireless Commun. Netw., vol. 2019, no. 1, pp. 197, Dec. 2019.

[2] E. Bjornson and L. Sanguinetti, "Scalable cell-free massive MIMO systems," IEEE Trans. Commun., vol. 68, no. 7, pp. 4247-4261, Jul. 2020.

[3] Y. Li and G. A. Aruma Baduge, "NOMA-aided cell-free massive MIMO systems," IEEE Wireless Commun. Lett., vol. 7, no. 6, pp. 950-953, Dec. 2018.

[4] X. Zhang and Q. Zhu, "NOMA and user-centric based cell-free massive MIMO over 6G big-data mobile wireless networks," GLOBECOM 2020 - 2020 IEEE Global Communications Conference, Taipei, Taiwan, 2020, pp. 1-6.

[5] M. Bashar, K. Cumanan, A. G. Burr, H. Q. Ngo, L. Hanzo and P. Xiao, "NOMA/OMA mode selection-based cell-free massive MIMO," Proc. IEEE ICC, pp. 1-6, May 2019.

[6] L. Dai, B. Wang, Y. Yuan, S. Han, I. Chih-lin and Z. Wang, "Nonorthogonal multiple access for 5G: solutions, challenges, opportunities, and future research trends," IEEE Commun. Magazine, vol. 53, no. 9, pp. 74-81, Sep. 2015.

[7] A. A. Ohashi et al., "Cell-free massive MIMO-NOMA systems with imperfect SIC and non-reciprocal channels," Early Access in IEEE Wireless Commun. Lett..

[8] E. Björnson, J. Hoydis and L. Sanguinetti, "Massive MIMO Networks: Spectral, Energy, and Hardware Efficiency," Now Foundations and Trends, 2017.
[9] J. Morte Palacios, O. Raeesi, A. Gokceoglu and M. Valkama, "Impact of channel non-reciprocity in cell-free massive MIMO," IEEE Wireless Commun. Lett., vol. 9, no. 3, pp. 344-348, Mar. 2020.

[10] H. Q. Ngo, A. Ashikhmin, H. Yang, E. G. Larsson and T. L. Marzetta "Cell-free massive MIMO versus small cells," IEEE Trans. Wireless Commun., vol. 16, no. 3, pp. 1834-1850, Mar. 2017.

[11] ETSI TR 38 901, "Study on channel model for frequencies from 0.5 to 100 GHz," 3GPP TR 38.901 version 16.1.0 Release 16, Dec. 2019. 